\title{
Sección Informativa
}

\section{CONGRESO INTERNACIONAL DE ENDOSCOPIA GINECOLOGICA Y MICROCIRUGIA}

(International Congress on Gynecologic Endoscopy and Microsurgery)

JERUSALEN. TEL-AVIV.

Israel. Febrero 15/22 1.980

Patrocinado por:

Soc. Obstetricia y Ginecología de Israel

Sociedad de Fertilidad de Israel

Escuela de Medicina de Hadassah

Fac. de Medicina Univ. Tel-Aviv.

Escuela de Medicina Beer-Sheba.

Colegio de Medicina Univ. de California. U.S.A.

Información: Jordan M. Phillips, M.D. 11239 So. Lakewood. Blvd DOWNEY,CA 90241.

Agentes de viajes:

Garber Travel. 1406 Beacon St.

Brookline Massachusetts 92146

\section{CONGRESO INTERNACIONAL DE SENOLOGIA}

(International Congress on Senology)

Aspectos interdiciplinarios en las enfermedades del seno.

HAMBURGO. (Alemania)

Mayo 27 a 31 de 1980

Temario: Enfermedades benignas y malignas del seno femenino.

Etiología.

Aspectos psicológicos

Endocrinología.

Histopatología.

Diagnóstico.

Pronóstico.

Tratamiento.

Información: Prof. Dr. H.J. Frischbier, Universitats Frauenklinik

Martinistrasse 52,2000 HAMBURG 20 . Germany.
Idiomas del Congreso: Inglés, Francés, y Alemán. (Traducción simultánea).

X. CONGRESO MUNDIAL DE GINECOLOGIA Y OBSTETRICIA

SAN FRANCISCO. Cal. EEUU.

Fecha: Septiembre 1982

Información: (Col) FECOLSOG. Ap. 14961.34188. Bogotá Col.

\section{CURSO DE POS-GRADO DE CITOPATO- LOGIA}

Baltimore. Maryland. Abril 14-25 1980.

Organizado por la Universidad de Johns Hopkins Fac. de Med. para patólogos certificados por el Am. Board of Pathology.

Información: John K. Frost, M.D. Pathology Building.

Johns Hopkins Hosp. BALTIMORE. 21205. USA.

Todo el curso será dado en Inglés.

\section{SOCIEDAD COLOMBIANA DE SEXO- LOGIA}

Ha sido constituída la Sociedad Colombiana de Sexología en la ciudad de Manizales (Col), sus finalidades son: a) Promover el estudio científico de todos los aspectos de la sexología. b) Servir de Foro interdisciplinario para la difusión del conocimiento sexológico. c) Defender la dignidad de la sexología como disciplina científica. d) Velar por el ejercicio idóneo de las actividades sexológicas profesionales.

Dirección: Dr. Heli Alzate. Apartado aéreo. 631. Manizales. Colombia. 